\title{
Gangrenous cholecystitis in male patients: A study of prevalence and predictive risk factors
}

\author{
Carlos Augusto Gomes ${ }^{1}$, Cleber Soares Junior ${ }^{1}$, Salomone Di Saverio ${ }^{2}$, Massimo Sartelli ${ }^{3}$, \\ Poliana Graciele de Souza Silva ${ }^{4}$, Agnes Silva Orlandi ${ }^{4}$, Thais Lacerda Heringer ${ }^{4}$, \\ Felipe Couto Gomes ${ }^{5}$, and Fausto Catena ${ }^{6}$
}

\begin{abstract}
${ }^{1}$ Surgery Department, Hospital Universitário Therezinha de Jesus, Faculdade de Ciências Médicas e da Saúde Juiz de Fora (SUPREMA), Hospital Universitário da Universidade Federal de Juiz de Fora (uffi), Juiz de Fora, Brazil, ${ }^{2}$ Cambridge University Hospitals NHS Foundation Trust, Addenbrooke's Hospital, Cambridge, England, ${ }^{3}$ Department of Surgery, Macerata University Hospital, Macerata, Italy, ${ }^{4}$ Internal Medicine Unit, Hospital Universitário da Universidade Federal de Juiz de Fora (uffi), Internal Medicine Unit, Hospital Universitário Therezinha de Jesus, Faculdade de Ciências Médicas e da Saúde Juiz de Fora (SUPREMA), Juiz de Fora, Brazil, ${ }^{6}$ Department of General Surgery, Maggiore Hospital, Parma, Italy and Department of Surgery, "Infermi" Hospital, Rimini, Italy
\end{abstract}

\begin{abstract}
Backgrounds/Aims: The prevalence and risk factors of gangrenous cholecystitis in male are unknown. Objective: To verify the prevalence and risk factors of gangrenous cholecystitis in males. Methods: This cross-sectional study includes 95 patients (59.5 \pm 17.1 years), with clinical and histopathological diagnosis, operated laparoscopically on 2012-2016. Eligibility was decided based on the variables of age; tachycardia ( $>100 \mathrm{bpm})$; leukocytosis $\left(>10,000 / \mathrm{mm}^{3}\right)$; Murphy's sign; gallbladder wall thickness $(>4 \mathrm{~mm}$ ); biochemical tests, morbidities (diabetes, alcoholism, smoking) and mortality. Multivariate regression, the chi-squared and Prevalent Chances Ratio (PCR) were used to define a few parameters. Results: The prevalence of gangrenous cholecystitis in men older than 50 years was $29.3 \%$. The risk factors for the disease were as follows: diabetes mellitus $(p=0.006, \mathrm{RCP}=4.191)$, leukocytosis $(p=0.003)$, gallbladder thickness greater than $4 \mathrm{~mm}(p=0.035, \mathrm{RCP}=3.818)$, which increased mortality $[(p=0.04)(\mathrm{RCP}=8.001)]$. Murphy's sign showed a negative association $(p=0.002, \mathrm{RCP}=0.204)$. Values close to significance were observed in relation to gamma glutamyl transferase $(p=0.083, \mathrm{RCP}=3.125)$ and hospital stay $(p=0.061, \mathrm{RCP}=2.765)$. Conclusions: Male gender, and age older than 50 years, were correlated with a high prevalence of necrosis, higher than that reported in females. The risk factors for developing necrosis are the same as those described for female patients. (Ann Hepatobiliary Pancreat Surg 2019;23:34-40)
\end{abstract}

Key Words: Abdominal infections; Abdominal surgery; Cholecystectomy; Complication; Laparoscopic surgery

\section{INTRODUCTION}

Gangrenous cholecystitis (GC) is defined as gallbladder necrosis and perforation because of progressive inflammation and infection, which impairs the arterial wall supply, leading to wall ischemia. It represents a serious complication of acute cholecystitis (AC), with an estimated prevalence of $2 \%$ to $20 \%$, following the advent of laparoscopy. A recent study, which evaluated 141,970 cholecystectomies, identified 7017 patients presenting with GC. The 30-day mortality and overall complications rates were $1.2 \%$ and $10.8 \%$, respectively. ${ }^{1-4}$

The pathophysiology of gallbladder wall inflammation involves impacted calculus in the infundibulum or the cystic duct, followed by secondary bacterial infection (Enterobacteriaceae) from splanchnic lymphocytes. ${ }^{5}$ These features allow AC diagnosis at different stages of severity, including local and diffuse complications (with or without organic dysfunction). ${ }^{4,5}$ Early diagnosis should be a constant priority to prevent sepsis or septic shock. In these

Received: May 31, 2018; Revised: July 21, 2018; Accepted: July 26, 2018

Corresponding author: Carlos Augusto Gomes

Surgery Department, Hospital Universitário Therezinha de Jesus, Faculdade de Ciências Médicas e da Saúde Juiz de Fora (SUPREMA), Hospital Universitário da Universidade Federal de Juiz de Fora (ufjf), Rua Senador Salgado Filho 510 / 1002, Bairro Bom Pastor, Juiz de Fora 36021-660, Brazil

Tel: +55-21-32-3218-3188, Fax: +55-32999450005, E-mail: caxiaogomes@terra.com.br

Copyright (C) 2019 by The Korean Association of Hepato-Biliary-Pancreatic Surgery

This is an Open Access article distributed under the terms of the Creative Commons Attribution Non-Commercial License (http://creativecommons.org/ licenses/by-nc/4.0) which permits unrestricted non-commercial use, distribution, and reproduction in any medium, provided the original work is properly cited. Annals of Hepato-Biliary-Pancreatic Surgery • pISSN: 2508-5778 • elSSN: 2508-5859 
cases, hemodynamic stabilization and antibiotics are warranted and operative treatment is mandatory.

Abdominal ultrasonography (USG) is the first-choice imaging modality for AC diagnosis. The findings of gallstones, gallbladder wall thickness and Murphy's sign on USG show a high predictive value for AC diagnosis (95\%). However, in the presence or clinical suspicion of complications or diagnostic uncertainty, abdominal computed tomography (CT) is recommended. ${ }^{6,7}$

Risk factors for the development of gallbladder necrosis may include advanced age, sex, fever, leukocytosis, increased C-reactive protein (CRP) levels, and thrombocytopenia. Biochemical abnormalities, total parenteral nutrition, fasting, diabetes mellitus (DM), coronary artery disease, chronic obstructive pulmonary disease (COPD), cardiovascular accident, trauma, obesity, thickening of the gallbladder wall and/or pericholecystic fluid detected by USG, impacted calculus in the infundibulum, and sepsis are other risk factors. ${ }^{8-14}$

Although the treatment of GC patients is similar to that of patients with $\mathrm{AC}$, increased risks of postoperative complications, morbidity and mortality were detected in the first group. Terho et al. ${ }^{4}$ identified age higher than 65 years, male sex, impaired renal function and conversion to laparotomic approach as risk factors for complications. ${ }^{4,15}$ It is also known that early operative treatment (24-72 h) is the best option in both conditions, although the latter is still the preferred choice up to 7 days after the onset of symptoms (acute illness). ${ }^{5,6}$

It is noteworthy that the findings were derived from studies reporting patients of both sexes, suggesting bias. The results were based on analyses with a greater proportion of women, and do not reflect the prevalence of the disease in males accurately. Thus, our research is unique in that it evaluated whether or not the prevalence and risk factors in the male sex were similar to those observed in current publications.

Male patients hospitalized with clinical and histopathological AC diagnosis were retrospectively studied to investigate the prevalence of $\mathrm{GC}$ and the associated risk factors. The results, which are still not fully clear, may contribute to treatment priorities and decision-making on $\mathrm{AC}$ in males, by reducing treatment time and possible complications.

The objective of this study was to investigate the preva- lence of GC and the risk factors in male patients hospitalized with clinical and pathological AC diagnosis and submitted to laparoscopic treatment.

\section{MATERIALS AND METHODS}

This is a cross-sectional and retrospective, exploratory study, carried out at Monte Sinai Hospital in Juiz de Fora, Minas Gerais (Brazil) in the period between 2012 and 2016. The protocol was approved by the Research Ethics Committee of the Federal University of Juiz de Fora (CEP/UFJF). The study did not interfere with any physical, psychological and social well-being of the patients, according to Resolution 196/96 of the National Health Council/Ministry of Health on research involving human beings.

The sampling was non-probabilistic and included 95 patients, with a mean age of $59.5 \pm 17.1$ years (median age, 61.0 years), subjected to laparoscopic cholecystectomy (LC) by the Digestive System Surgery Service. The patient sample strictly met the criteria and the statistical needs of the study, with a sample error of $\pm 4.5 \%$. Three individuals were excluded due to the complete absence of data in their medical records.

Only male patients with clinical and pathological AC diagnosis were included in the study. Data extraction from medical records was carried out by three researchers from the Federal University of Juiz de Fora (UFJF) Medical School.

The prevalence and predictive risk factors of GC in men were estimated. The defined variables were as follows: age; tachycardia ( $>100 \mathrm{bpm}$ ); presence of Murphy's sign; gallbladder wall thickness $(>4 \mathrm{~mm})$; laboratory tests: [alanine amino transferase $(\mathrm{ALT})=38.9 \mathrm{U} / \mathrm{L}$, gamma-glutamyl-transpeptidase $(\mathrm{GGT})=44 \mathrm{U} / \mathrm{L}]$; leukocytosis $\left(>10,000 / \mathrm{mm}^{3}\right)$; associated morbidities (DM, alcoholism, smoking); and mortality.

\section{Statistical analysis}

The variables under investigation were divided into continuous quantitative and qualitative dichotomies. A descriptive and exploratory statistical study was performed using absolute frequencies (n), relative frequencies (\%), measures of central tendency (mean), and measures of dispersion (standard deviation). The analysis of the com- 
parative characteristics of the dichotomous qualitative variables was conducted using $2 \times 2$ contingency tables, containing the absolute (n) and relative (\%) frequencies. A Chi-Squared Test of Independence was performed to determine the association between the variables. The level of significance for this test was a $95 \%$ confidence interval $(p$-value $\leq 0.05)$. To measure prevalence, we used the prevalence odds ratio (RCP), especially in order to express the relationship between the probabilities of an exposed individual carrying the condition of interest, when compared with non-exposed individuals.

Multivariate binary logistic regression was used to analyze all the variables, with and without statistical significance in the univariate analysis. Therefore, the results in the analysis were divided into two groups according to their significance. The first group included variables with significance and the second group contained variables clinically important for the prevalence of disease, which did not show a significant effect. The Statistical Software SPSS version 21.0 was used for statistical evaluation and database assembly.

\section{RESULTS}

Table 1 displays the behavior of the variables studied by univariate analysis. Among the patients who developed gallbladder necrosis, 95.5\% manifested leukocytosis; 59.3\% presented with positive Murphy's sign; $67.7 \%$ showed gallbladder wall thickening $>4 \mathrm{~mm}$ and $11.1 \%$ died. Concurrently, $53.3 \%$ of the patients showed GGT greater than $45 \mathrm{U} / \mathrm{L}$ and $69.2 \%$ were hospitalized for more than 24 hours.

The results of the multivariate logistic evaluation are displayed in Table 2. Nearly one-third (29.3\%, $n=27)$ of the patients were diagnosed with $\mathrm{GC}$, and all were greater than 50 years of age. It was also observed that the prevalence of gallbladder necrosis was significant in diabetic patients $(p=0.006)$, an incidence 4.1 times higher than in non-diabetics $(\mathrm{RCP}=4.191, \mathrm{CI}=1.429-12.292)$.

Similarly, the relationship between GC and gallbladder wall thickness $>4 \mathrm{~mm}$ was significant $(p=0.035)$. It resulted in 3.8-fold greater probability of GC compared with patients showing a thickness $<4 \mathrm{~mm}(\mathrm{RCP}=3.818, \mathrm{CI}=$ 1.032-14.122). When evaluating the presence of leukocytosis, we found that patients with counts greater than $10,000 / \mathrm{mm}^{3}$ presented with a significant risk $(p=0.003)$ of
Table 1. Variables in male patients with clinical and histological diagnosis of acute cholecystitis treated with laparoscopic cholecystectomy $(n=92)$

\begin{tabular}{|c|c|c|}
\hline & Frequency (n) & Percentage $(\%)$ \\
\hline \multicolumn{3}{|l|}{ Necrosis } \\
\hline Yes & 27 & 29.3 \\
\hline No & 65 & 70.7 \\
\hline \multicolumn{3}{|c|}{ Hospital stay } \\
\hline$<24 \mathrm{~h}$ & 8 & 30.8 \\
\hline$>24 \mathrm{~h}$ & 18 & 69.2 \\
\hline \multicolumn{3}{|c|}{ Diabetes mellitus } \\
\hline Yes & 10 & 37.0 \\
\hline No & 17 & 63.0 \\
\hline \multicolumn{3}{|l|}{ Leukocytes } \\
\hline$>10.000$ & 21 & 95.5 \\
\hline$<10.000$ & 1 & 4.5 \\
\hline \multicolumn{3}{|l|}{ Alcoholism } \\
\hline Yes & 3 & 11.1 \\
\hline No & 24 & 88.9 \\
\hline \multicolumn{3}{|l|}{ Smoking } \\
\hline Yes & 3 & 11.1 \\
\hline No & 24 & 88.9 \\
\hline \multicolumn{3}{|c|}{ Murphy's sign } \\
\hline Present & 16 & 59.3 \\
\hline Absent & 11 & 40.7 \\
\hline \multicolumn{3}{|c|}{ Heart rate (beat/min) } \\
\hline$<100$ & 24 & 88.9 \\
\hline$>100$ & 3 & 11.1 \\
\hline \multicolumn{3}{|l|}{ ALT } \\
\hline$<38.9$ & 12 & 60.0 \\
\hline$>39.0$ & 8 & 40.0 \\
\hline \multicolumn{3}{|l|}{ GGT } \\
\hline$<44.0$ & 7 & 46.7 \\
\hline$>45.0$ & 8 & 53.3 \\
\hline \multicolumn{3}{|l|}{ Death } \\
\hline Yes & 3 & 11.1 \\
\hline No & 24 & 88.9 \\
\hline
\end{tabular}

$\overline{\text { ALT, alanine aminotransferase; GGT, gamma-glutamyltrans- }}$ peptidase

GC. Mortality was another significant variable in this series. There were $3(11.1 \%)$ deaths in GC patients, compared with $1(1.5 \%)$ in those without GC. Therefore, the GC patients carried 8-fold greater probability of death (RCP=8.001, $p=0.040$ ) (Table 2).

The presence of Murphy's sign showed a negative correlation with GC $(p=0.002)$. Thus, the probability of a non-GC patient presenting with positive Murphy's sign was approximately $80 \%$ lower than in patients with GC ( $\mathrm{RCP}=0.204, \mathrm{CI}=0.070-0.593)$.

Biochemically, a GGT below $44 \mathrm{U} / \mathrm{L}$ was observed in 7 (46.7\%) GC patients with non-GC, compared with 7 patients 
Table 2. Analysis of variables with significance according to the study design $(n=92)$

\begin{tabular}{|c|c|c|c|c|c|c|c|c|}
\hline & \multicolumn{2}{|c|}{ Necrosis present } & \multicolumn{2}{|c|}{ Necrosis absent } & \multirow{2}{*}{ Total } & \multirow{2}{*}{$p$-value } & \multirow{2}{*}{$\mathrm{RCP}$} & \multirow{2}{*}{ CI $95 \%$} \\
\hline & $\mathrm{n}^{1}$ & $\%$ & $\mathrm{n}^{1}$ & $\%$ & & & & \\
\hline \multicolumn{9}{|c|}{ Diabetes mellitus } \\
\hline Yes & 10 & 37.0 & 8 & 12.3 & 18 & $0.006^{*}$ & 4.191 & $1.429-12.292$ \\
\hline No & 17 & 63.0 & 57 & 87.7 & 74 & & & \\
\hline \multicolumn{9}{|l|}{ Thickness } \\
\hline$>4 \mathrm{~mm}$ & 24 & 88.9 & 44 & 67.7 & 68 & $0.035^{*}$ & 3.818 & $1.032-14.122$ \\
\hline$<4 \mathrm{~mm}$ & 3 & 11.1 & 21 & 32.3 & 24 & & & \\
\hline \multicolumn{9}{|c|}{ Hospital stay } \\
\hline$<24 \mathrm{~h}$ & 8 & 30.8 & 9 & 13.8 & 17 & $0.061 * *$ & 2.765 & $0.929-8.229$ \\
\hline$>24 \mathrm{~h}$ & 18 & 69.2 & 56 & 86.1 & 74 & & & \\
\hline \multicolumn{9}{|l|}{ Alcoholism } \\
\hline Yes & 3 & 11.1 & 14 & 21.5 & 17 & 0.241 & 0.455 & $0.119-1.736$ \\
\hline No & 24 & 88.9 & 51 & 78.5 & 75 & & & \\
\hline \multicolumn{9}{|l|}{ Smoking } \\
\hline Yes & 3 & 11.1 & 11 & 16.9 & 14 & 0.480 & 0.614 & $0.157-2.401$ \\
\hline No & 24 & 88.9 & 54 & 83.1 & 78 & & & \\
\hline \multicolumn{9}{|l|}{ Murphy sign } \\
\hline Present & 16 & 59.3 & 57 & 87.7 & 73 & $0.002 *$ & 0.204 & $0.070-0.593$ \\
\hline Absent & 11 & 40.7 & 8 & 12.3 & 19 & & & \\
\hline \multicolumn{9}{|l|}{ ALT } \\
\hline$<38.9$ & 12 & 60.0 & 28 & 56.0 & 40 & 0.760 & 1.179 & $0.411-3.383$ \\
\hline$>39.0$ & 8 & 40.0 & 22 & 44.0 & 30 & & & \\
\hline \multicolumn{9}{|l|}{ GGT } \\
\hline$<44.0$ & 7 & 46.7 & 7 & 21.9 & 14 & $0.083 * *$ & 3.125 & $0.838-11.649$ \\
\hline$>45.0$ & 8 & 53.3 & 25 & 78.1 & 33 & & & \\
\hline \multicolumn{9}{|l|}{ Heart rate } \\
\hline$<99$ & 24 & 88.9 & 62 & 95.4 & 86 & 0.251 & 0.387 & $0.073-2.093$ \\
\hline$>100$ & 3 & 11.1 & 3 & 4.6 & 6 & & & \\
\hline \multicolumn{9}{|c|}{ Leucocytes (/mcL) } \\
\hline$>10000$ & 21 & 95.5 & 33 & 61.1 & 54 & $0.003 *$ & - & - \\
\hline$<10000$ & 1 & 4.5 & 21 & 38.9 & 22 & & & \\
\hline \multicolumn{9}{|l|}{ Death } \\
\hline Yes & 3 & 11.1 & 1 & 1.5 & 4 & $0.040^{*}$ & 8.001 & $0.793-80.699$ \\
\hline No & 24 & 88.9 & 64 & 98.5 & 88 & & & \\
\hline
\end{tabular}

Thickness, gallblader wall thickness; AST, aspartate aminotransferase; ALT, alanine aminotransferase; GGT, gamma-glutamyl transpeptidase; RCP, prevalence odds ratio

*significant values HR (Heart rate)

**strongly significant

$(21.9 \%)$ in the $\mathrm{GC}$ group $(\mathrm{RCP}=3.125, \mathrm{CI}=0.838-11.649$, $p=0.083$ ) a value close to significance. Thus, the probability of a GC patient presenting with GGT elevation was 3.1-fold higher than non-GC. The other biochemical tests (AST, ALT) did not show significance among patients with AC, with or without gangrene (Table 2). The hospitalization time also showed a $p$-value close to significance $(p=0.061)$ because it depended on the severity of the disease. Thus, patients diagnosed with GC showed a 2.7-fold higher probability of hospitalization for an extended period $(\mathrm{RCP}=2.765, \mathrm{CI}=0.929-8.229)$.
The heart rate (HR) did not show a significant association with GC $(p=0.25)$. Thus, the likelihood of a non-GC patient manifested a $\mathrm{HR}$ of fewer than $99 \mathrm{bpm}$ is $60 \%$ higher than in patients with $\mathrm{GC}(\mathrm{RCP}=0.387, \mathrm{CI}=0.073-2.093)$. In the analysis of the other associated morbidities (alcoholism and smoking), no significant differences were observed ( $p=0.24$ and $p=0.48$, respectively).

\section{DISCUSSION}

Our investigations showed that for the 95 male patients 
aged above 50 years and admitted to the institution with confirmed diagnosis of $\mathrm{AC}$ based on the anatomical and pathology examination, the prevalence of GC was $29.3 \%$, ie, one-third of all hospitalized patients. The study unraveled unknown demographic and epidemiological data of the disease. Further, this information is fundamental to the emergency management personnel, who provide the first care to the patients. It highlights the possible complications and scope for therapeutic intervention, in line with current surgical recommendations (at up to 72 hours). Ultimately, it can reduce postoperative complications and deaths. ${ }^{6}$

Gallbladder necrosis has been diagnosed with a higher prevalence in men. ${ }^{1,9,16,17}$ Men are considered to have a higher pain threshold and tend to seek delayed medical attention. Further, repeated undiagnosed subacute inflammation causing anatomical changes in the gallbladder may predispose to complications. ${ }^{16,17}$

The real impact of each variable or its association with the possibility of vesicular necrosis was hard to assess, due to methodological failures observed in most studies. These failures are in principle conceptual, because the studies represent retrospective database analyses. Moreover, the study highlights the need to define 'leukocytosis', 'HR' and 'histological confirmation' more precisely (ie, 'lack of histological confirmation'), which justify the heterogeneity of the results involving different series. Although bias remains, it differs from those of other studies because, for the first time, the results are derived exclusively from male patients.

The significant risk factors of GC in this series were as follows: diabetes mellitus, gallbladder thickness $>4$ $\mathrm{mm}$, elevation of GGT and leukocytosis (Table 2). On the other hand, the presence of Murphy's sign was a protective factor against gallbladder necrosis. Therefore, the combined presence of these factors is a source of confusion for the surgeon, suggesting "disease is observed with the scalpel in hand" since gallbladder necrosis may be already present when the patient is admitted to hospital.

Other authors also refer to age greater 45-50 years $^{1,3,7,12,18-20}$ and male sex ${ }^{1,3,7,12,18,20}$ as risk factors for the development of gallbladder necrosis. The study of Yacoub et al., although faced with the same problems, is interesting and, similar to the present study, easy to apply at the bedside. They developed a score to stratify patients with AC into groups with high, intermediate, or low probability of GC, based on five aspects: male sex, age $>45$ years, HR $>90$ beats $/ \mathrm{min}$, leukocytosis $>13,000 / \mathrm{mm}^{3}$ and USG gallbladder wall thickness $>4.5 \mathrm{~mm}$. They found that patients presenting with all the listed factors presented with a marked risk of gallbladder necrosis (87\%). Those presenting with 3 to 4 factors showed an intermediate risk of necrosis $(33 \%)$. They concluded that the system prioritized patients for emergency cholecystectomy based on the expected pathology. ${ }^{12}$ These findings corroborate those of our study correlating male sex and age $<50$ years as an intermediate risk factor for gallbladder necrosis, warranting urgent operative treatment.

A review of 5,812 patients undergoing cholecystectomy including 2,219 with AC and 351 with GC, found a low surgical ability to diagnose GC preoperatively, including only $9 \%$ of cases. However, patients aged $>45$ years, HR $>90$ bpm, leukocyte count $>13,000 / \mathrm{mm}^{3}$, gallbladder wall thickness $>4 \mathrm{~mm}$ and presence of pericolic fluid in USG, American Society of Anesthesiology (ASA) score $>2$, without regard to sex, presented with a $63 \%$ probability of GC when all factors were present. ${ }^{16}$

In contrast, Ganapathi et al. ${ }^{2}$ did not identify advanced age as a risk factor in the evolution from AC to GC. In fact, the conceptual bias was apparent in the evaluation of this and the following variables. Patients aged 45-50 years are not elderly, and the studies referenced above included this age group as a cutoff point. Perhaps the most adequate proposition is to define patients aged over 60-65 years as advanced age and controlling for this criterion in subsequent investigation.

The presence of DM showed a significant correlation with the prevalence of GC in male patients hospitalized with AC (4.1-fold higher probability). Other authors also demonstrated the same correlation. ${ }^{2,11,19}$ One possible explanation involves changes in splanchnic vascularization secondary to the reduction of nociception due to diabetic neuropathy, impairing clinical manifestation and its early diagnosis. The issue is still controversial, as other highlighted studies have not confirmed this correlation. ${ }^{12,13,16,20}$ Thus, while the controversy persists, such patients warrant prompt evaluation and surgical intervention to diminish the risk of vesicular necrosis. In addition, a prospective multi-institutional study with appropriate methodology and agreed definitions of the parameters operating under 
the seal of a respected Surgical Society may provide valuable insights.

The thickness of the USG vesicular wall $(>4-4.5 \mathrm{~mm})$ combined with the presence of impacted biliary calculus in the infundibulum and their association with GC risk is still uncertain. Occasionally, a USG alteration is predictive of $\mathrm{AC}$, without necessarily indicating vesicular necrosis. $^{21}$ In addition, treatment and prognosis may be delayed by such USG changes. ${ }^{9}$ In the present study, the parameter demonstrated a significant relationship ( $p=$ 0.035 ) and added 3.8-fold probability to the evolution of GC and therefore, warrant consideration and evaluation via robust studies. ${ }^{11,12,19,22,23}$

Leukocytosis was another significant risk factor for GC ( $p=0.003$ ), and $95 \%$ of GC patients had a leukocyte count greater than $10,000 / \mathrm{mm}^{3}$. The study of the variable provides favorable $\mathrm{e}^{1,18}$ and unfavorable $\mathrm{e}^{19,21,22}$ evidence. However, the differences in the definition of leukocytosis between the studies are the most important aspects. Thus, if leukocytosis is defined as a leukocyte count above $10,000 / \mathrm{mm}^{3}$, results may vary from studies involving definitions with counts above $13,000 / \mathrm{mm}^{3}$. These definitions require standardization as well. ${ }^{22}$

Smoking and alcoholism are parameters that have yet to be correlated with gallbladder necrosis. However, Ganapathi et al. ${ }^{2}$ concluded that both factors were significant in the development of vesicular necrosis. Despite analytical discrepancies, since the study includes only males affected with AC, the low prevalence of these associated morbidities may have interfered with the result.

Finally, another significant finding was the positive correlation between $\mathrm{GC}$ and death rate, which increased 8 -fold $(p=0.040)$. This result is in line with other published results, which also found an increase in GC-related mortality, when compared with AC without necrosis. ${ }^{19,20}$ The study included only patients classified under grade two or three according to Tokyo guidelines. However, we were not interested in stratifying them, because our main objective was the gangrenous event. The stratification of patients will enrich the study, especially in the evaluation of mortality, which occurred in three (about 11\%) complicated cases. The first patient was a male in his 80 s and diagnosed with diffuse peritonitis secondary to gallbladder perforation. The second patient was another octogenarian operated for septic shock. A retrospective analysis may lead to discussion of the importance of cholecystostomy in these critical patients. The procedure is the now the first choice in the management of such patients. The last patient died from a progressive sepsis after necrotic empyema of gallbladder with massive contamination of superior left quadrant. In contrast, other investigators failed to demonstrate a correlation between GC and death. However, the findings do not suggest increased incidence of complications when patients undergo treatment at specialized units. $^{11}$

\section{ACKNOWLEDGEMENTS}

This manuscript represents the final requirement to obtain the Title of Scientific Initiation of Surgical Department of Universidade Federal de Juiz de Fora (ufjf), Ministry of Education, Minas Gerais, Brazil.

\section{REFERENCES}

1. Hunt DR, Chu FC. Gangrenous cholecystitis in the laparoscopic era. Aust N Z J Surg 2000;70:428-430.

2. Ganapathi AM, Speicher PJ, Englum BR, Perez A, Tyler DS, Zani S. Gangrenous cholecystitis: a contemporary review. J Surg Res 2015;197:18-24.

3. Di Saverio S. Emergency laparoscopy: a new emerging discipline for treating abdominal emergencies attempting to minimize costs and invasiveness and maximize outcomes and patients' comfort. J Trauma Acute Care Surg 2014;77:338-350.

4. Terho PM, Leppäniemi AK, Mentula PJ. Laparoscopic cholecystectomy for acute calculous cholecystitis: a retrospective study assessing risk factors for conversion and complications. World J Emerg Surg 2016;11:54.

5. Yokoe M, Takada T, Strasberg SM, Solomkin JS, Mayumi T, Gomi H, et al:; Tokyo Guidelines Revision Committee. TG13 diagnostic criteria and severity grading of acute cholecystitis (with videos). J Hepatobiliary Pancreat Sci 2013;20:35-46.

6. Fagan SP, Awad SS, Rahwan K, Hira K, Aoki N, Itani KM, et al. Prognostic factors for the development of gangrenous cholecystitis. Am J Surg 2003;186:481-485.

7. Knab LM, Boller AM, Mahvi DM. Cholecystitis. Surg Clin North Am 2014;94:455-470.

8. Önder A, Kapan M, Ülger BV, Oğuz A, Türkoğlu A, Uslukaya Ö. Gangrenous cholecystitis: mortality and risk factors. Int Surg 2015;100:254-260.

9. Yeh DD, Cropano C, Fagenholz P, King DR, Chang Y, Klein EN, et al. Gangrenous cholecystitis: deceiving ultrasounds, significant delay in surgical consult, and increased postoperative morbidity! J Trauma Acute Care Surg 2015;79:812-816.

10. Mok KW, Reddy R, Wood F, Turner P, Ward JB, Pursnani KG, et al. Is $\mathrm{C}$-reactive protein a useful adjunct in selecting patients for emergency cholecystectomy by predicting severe/gangrenous cholecystitis? Int J Surg 2014;12:649-653.

11. Nikfarjam M, Niumsawatt V, Sethu A, Fink MA, Muralidharan V, Starkey G, et al. Outcomes of contemporary management of gangrenous and non-gangrenous acute cholecystitis. HPB (Oxford) 
2011;13:551-558.

12. Yacoub WN, Petrosyan M, Sehgal I, Ma Y, Chandrasoma P, Mason RJ. Prediction of patients with acute cholecystitis requiring emergent cholecystectomy: a simple score. Gastroenterol Res Pract 2010;2010:901739.

13. Falor AE, Zobel M, Kaji A, Neville A, De Virgilio C. Admission variables predictive of gangrenous cholecystitis. Am Surg 2012; 78:1075-1078.

14. Kabul Gurbulak E, Gurbulak B, Akgun IE, Duzkoylu Y, Battal M, Fevzi Celayir M, et al. Prediction of the grade of acute cholecystitis by plasma level of C-reactive protein. Iran Red Crescent Med J 2015;17:e28091.

15. Dhir T, Schiowitz R. Old man gallbladder syndrome: gangrenous cholecystitis in the unsuspected patient population. Int J Surg Case Rep 2015;11:46-49.

16. Wu B, Buddensick TJ, Ferdosi H, Narducci DM, Sautter A, Setiawan L, et al. Predicting gangrenous cholecystitis. HPB (Oxford) 2014;16:801-806.

17. Ambe PC, Köhler L. Is the male gender an independent risk factor for complication in patients undergoing laparoscopic cholecystectomy for acute cholecystitis? Int Surg 2015;100:854-859.
18. Merriam LT, Kanaan SA, Dawes LG, Angelos P, Prystowsky JB, Rege RV, et al. Gangrenous cholecystitis: analysis of risk factors and experience with laparoscopic cholecystectomy. Surgery 1999; 126:680-685; discussion 685-686.

19. Borzellino G, Steccanella F, Mantovani W, Genna M. Predictive factors for the diagnosis of severe acute cholecystitis in an emergency setting. Surg Endosc 2013;27:3388-3395.

20. Bourikian S, Anand RJ, Aboutanos M, Wolfe LG, Ferrada P. Risk factors for acute gangrenous cholecystitis in emergency general surgery patients. Am J Surg 2015;210:730-733.

21. Kawai R, Hata J, Manabe N, Imamura H, Iida A, Nakatou R, et al. Contrast-enhanced ultrasonography with Sonazoid for diagnosis of gangrenous cholecystitis. J Med Ultrason (2001) 2016; 43:193-199.

22. Gomes CA, Junior CS, Di Saverio S, Sartelli M, Kelly MD, Gomes CC, et al. Acute calculous cholecystitis: review of current best practices. World J Gastrointest Surg 2017;9:118-126.

23. Khan ML, Abbassi MR, Jawed M, Shaikh U. Male gender and sonographic gall bladder wall thickness: important predictable factors for empyema and gangrene in acute cholecystitis. J Pak Med Assoc 2014;64:159-162. 